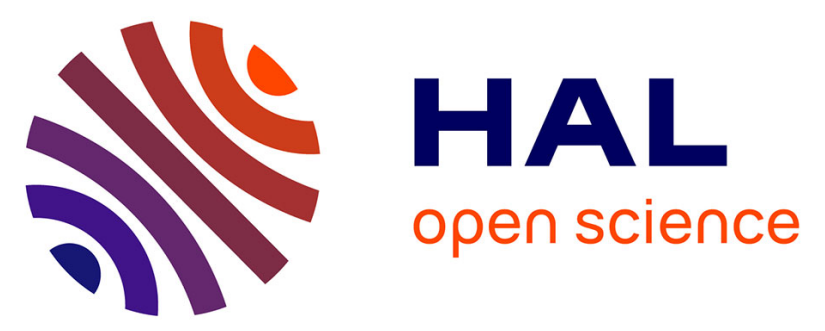

\title{
Follicular dendritic cells in follicular lymphoma and types of non-Hodgkin lymphoma show reduced expression of CD23, CD35 and CD54 but no association with clinical outcome
}

Mi Kyung Jin, Eva Hoster, Martin Dreyling, Michael Unterhalt, Wolfgang Hiddemann, Wolfram Klapper

\section{To cite this version:}

Mi Kyung Jin, Eva Hoster, Martin Dreyling, Michael Unterhalt, Wolfgang Hiddemann, et al.. Follicular dendritic cells in follicular lymphoma and types of non-Hodgkin lymphoma show reduced expression of CD23, CD35 and CD54 but no association with clinical outcome. Histopathology, 2011, 58 (4), pp.586. 10.1111/j.1365-2559.2011.03779.x . hal-00623321

\section{HAL Id: hal-00623321 \\ https://hal.science/hal-00623321}

Submitted on 14 Sep 2011

HAL is a multi-disciplinary open access archive for the deposit and dissemination of scientific research documents, whether they are published or not. The documents may come from teaching and research institutions in France or abroad, or from public or private research centers.
L'archive ouverte pluridisciplinaire HAL, est destinée au dépôt et à la diffusion de documents scientifiques de niveau recherche, publiés ou non, émanant des établissements d'enseignement et de recherche français ou étrangers, des laboratoires publics ou privés. 


\section{Histopathology}

\section{Follicular dendritic cells in follicular lymphoma and types of non-Hodgkin lymphoma show reduced expression of CD23, CD35 and CD54 but no association with clinical outcome}

\begin{tabular}{|c|c|}
\hline Journal: & Histopathology \\
\hline Manuscript ID: & HISTOP-12-09-0698.R1 \\
\hline Manuscript Type: & Original Article \\
\hline $\begin{array}{r}\text { Date Submitted by the } \\
\text { Author: }\end{array}$ & 26-Mar-2010 \\
\hline Complete List of Authors: & $\begin{array}{l}\text { Jin, Mi Kyung; Universityhospital Schleswig-Holstein, Campus Kiel, } \\
\text { Department of Pathology, Haematopathology Section and Lymph } \\
\text { Node Registry } \\
\text { Hoster, Eva; University of Munich, Department of Internal Medicine } \\
\text { III } \\
\text { Dreyling, Martin; University of Munich, Department of Internal } \\
\text { Medicine III } \\
\text { Unterhalt, Michael; University of Munich, Department of Internal } \\
\text { Medicine III } \\
\text { Hiddemann, Wolfgang; University of Munich, Department of } \\
\text { Internal Medicine III } \\
\text { Klapper, Wolfram; Universityhospital Schleswig-Holstein, Campus } \\
\text { Kiel, Department of Pathology, Hematopathology Section and } \\
\text { Lymph Node Registry }\end{array}$ \\
\hline Keywords: & follicular dendritic cells, follicular lymphoma, digital image analysis \\
\hline
\end{tabular}

\section{今 ScholarONE" \\ Manuscript Central}


Follicular dendritic cells in follicular lymphoma and types of non-Hodgkin lymphoma show reduced expression of CD23, CD35 and CD54 but no association with clinical outcome

Mi Kyung Jin ${ }^{1}$, Eva Hoster ${ }^{2}$, Martin Dreyling ${ }^{2}$, Michael Unterhalt ${ }^{2}$, Wolfgang Hiddemann ${ }^{2}$ and Wolfram Klapper ${ }^{1}$

${ }^{1}$ Department of Pathology, Hematopathology Section and Lymph Node Registry, University Hospital Schleswig-Holstein, Campus Kiel, Germany

${ }^{2}$ Department of Internal Medicine III, University of Munich, Germany

Keywords: follicular dendritic cells, follicular lymphoma, digital image analysis, CD23, CD35, CD54.

Running head: Follicular dendritic cells in NHL

Abstract words: 197

Text words: 2991 


\begin{abstract}
Aims: Follicular dendritic cells (FDC) are specialized antigen-presenting cells exclusively found in the germinal center (GC) which can be detected in B-cell nonHodgkin lymphomas (NHL) as reactive bystander cells. Recently, gene expression profiling revealed that FDC networks might be associated with the clinical outcome in follicular lymphoma. Herein we aimed to characterize FDC in NHL and to evaluate a possible association with outcome in follicular lymphoma.
\end{abstract}

Methods and Results: We determined semi-quantitatively the extent and the immunophenotype of FDC in reactive GC and NHL (follicular lymphoma, angioimmunoblastic T-cell lymphoma, mantle cell lymphoma) using fluorescencedouble staining and digital image analysis. In all NHL tested, CD23 and CD35 and CD54 were expressed at relatively low levels on FDC, comparable to FDC found in the dark zone of the GC. However, the extent of FDC networks did not correlate with the clinical outcome of 102 patients with follicular lymphomas treated within a prospectively randomized trial.

Conclusions: We conclude that FDC found in different types of NHL show quantitatively reduced expression of several proteins suggestive for functional differences between FDC in normal GC and NHL. The extent of the FDC networks in follicular lymphoma is not useful as a prognostic marker. 


\section{Introduction}

Follicular dendritic cells (FDC) represent a unique population of nonlymphoid accessory cells exclusively found in the germinal centers (GC) ${ }^{1-3}$. Within the GC, FDC form a cellular network that sustains the architecture of lymphoid follicles, and interact with the germinal center $B$ cells via several mechanisms involving adhesion molecules like CD54, FC-binding proteins like CD23 and complement receptors (CR) like CD35 ${ }^{1,2}$. The ability of FDC to trap and retain antigens in the form of immune complexes (IC) for long periods of time and the interaction between FDC and B cells are assumed to play a pivotal role in the selection of antigen specific $B$ cells and their clonal expansion in the GC ${ }^{4,5}$. Even though the fundamental biology and behavior of FDC have not yet been defined, it seems very likely that the integrity of FDC networks not only significantly influences GC reactions, but also plays a central role in the pathogenesis of several diseases ${ }^{6}$.

In certain B-cell non-Hodgkin lymphomas (NHL) FDC can be detected among the reactive bystander cells of the tumor. Whereas in marginal zone lymphomas the FDC are considered to represent remnants of GC that have been colonized by lymphoma cells, it has been suggested that FDC in mantle cell lymphoma (MCL), follicular lymphoma (FL) and angioimmunoblastic T-NHL (AITL) are newly generated during lymphoma growth and expansion ${ }^{7}$. In lymphomas structural alterations such as an atypical shape or expansion of the FDC networks are frequently detectable and are used as diagnostic features for the histopathological lymphoma diagnosis. The immunophenotype of FDC in lymphomas has been characterized, and reduced expression of markers of mature FDC was noted in $\mathrm{FL}^{8,9}$. However, the few reports about FDC immunophenotypes in lymphomas have used conventional immunohistochemical staining methods, light microscopy and eyeballing. Since FDC are large cells with long dendritic extensions and hardly detectable cell bodies, the quantification of the extent of FDC networks or the immunophenotype of FDC using conventional staining, light microscopy and eyeballing is highly observer dependent. Recently, gene expression profiling, a quantitative method, revealed expression of FDC related genes to be associated with the clinical course in follicular lymphoma ${ }^{10}$. Since gene expression profiling is not applicable to formalin-fixed, paraffinembedded tissue (FFPE), alternative methods to quantify bystander cells e.g. by immunohistochemistry might be useful for clinical application. 
In this study we developed a method employing multicolor immunofluorescence staining followed by digital image analysis to detect FDC in paraffin embedded tissue. Our aims were (i) to characterize differences and similarities between the FDC immunophenotypes in reactive $\mathrm{GC}$ and $\mathrm{NHL}$ in order to gain insights into a possible role of FDC in lymphoma genesis and (ii) to quantify the extent of FDC networks precisely and to correlate the extent of FDC networks with clinical outcome in FL. 


\section{Methods and Materials}

\section{Tisssue samples}

Formalin-fixed, paraffin-embedded tissue samples from representative examples of tonsils $(n=10)$, follicular lymphoma grade 1 or $2(n=5)$, mantle cell lymphoma, classical type ( $n=7)$, and angioimmunoblastic T-cell lymphoma $(n=5)$ were selected from the files of the Lymph Node Registry in Kiel. The lymphoma biopsy specimens were anonymized according to the ethical guidelines of the Medical Faculty of the University of Kiel (institutional review board) for retrospective analysis of archived material. All cases were classified according to the WHO classification by means of morphologic examination and immunophenotyping on paraffin sections ${ }^{7}$.

For clinical correlation 108 formalin-fixed, paraffin-embedded lymph node biopsy specimens from patients with advanced stage follicular lymphoma recruited between May 1996 and December 2000 for the trial "CHOP versus MCP" of the German LowGrade Lymphoma Study Group (GLSG) were analyzed ${ }^{11}$. In this study only primary biopsies obtained before any treatment for advanced stage FL were analyzed. All studies were performed on full sections of the biopsy specimen. For analysis of patient material from the GLSG trials informed consent of the patients is available.

\section{Flourescence double staining}

Four-micrometer thick sections of the tonsil or lymph node tissues were mounted on 3-aminopropyltriethoxy silane (APES)-coated slides and deparaffinized. For double staining, Ki-M4p, a mouse monoclonal antibody detecting all FDC (pan-FDC marker, undiluted cell culture supernatant ${ }^{12}$ ), was combined with one of the following antibodies: rabbit anti-human CD23 (Neomarkers, Freemont, CA, USA, diluted 1:50), rabbit anti-human CD35 (Santa Cruz Biotechnology, Santa Cruz, CA, USA diluted 1:50) or goat anti-human CD54 (R\&D Systems, Minneapolis, MN, USA, diluted 1:50).

Several antigen retrieval methods were tested to obtain reliable staining for each marker. In the final protocol, the slides with tissue sections to be used for double staining were boiled in citric acid buffer at $\mathrm{pH} 3.0$ for 4 minutes in a pressure cooker. This procedure yielded staining patterns for each antibody that were comparable to single staining. 
The extent of FDC networks in study samples to be used for clinical correlation was assessed by single staining using Ki-M4p or CD23. For this purpose, the epitopes were unmasked by digesting the sections with protease $\mathrm{K}$ at $\mathrm{pH} 7.5$ for 20 minutes or by boiling the sections in citric acid butter at $\mathrm{pH} 6$ for 3 minutes in a pressure cooker.

After the antigen retrieval, the slides were transferred without delay to cold running water for washing and then immersed in PBS buffer. Antibody incubation was performed overnight in a moist chamber at room temperature. The staining protocols were carefully established to avoid unspecific staining of cells other than FDC. Matching fluorescence labeled secondary antibodies (Alexa Fluor 488 or Alexa Fluor 594, Invitrogen, Carlsbad, CA, USA) were incubated on the sections in a moist chamber for 60 minutes at room temperature. Afterwards, the sections were thoroughly washed with PBS and manually covered using Fluorescent Mounting Medium.

\section{Digital image analysis}

In every section, five independent areas containing a GC in tonsils, a malignant follicle in FL or areas with FDC networks in MCL or AITL were selected and photographed at a magnification of 200x (Axioscope 40 microscope Zeiss, Jena, Germany, equipped with an Image View Camera, Soft Imaging Systems Olympus, Hamburg, Germany) at room temperature. To ensure that exclusively areas with FDC were analyzed, the GC or FDC-containing areas were cut out of the pictures by means of Adobe Photoshop 7.0 and pasted into a separate blank image with a white background. The dark zone of the GC can easily be identified because of the reduced FDC network and was isolated from the rest of the GC in the same way. All images were analyzed using the software analySIS (Soft Imaging Systems Olympus, Hamburg, Germany). For this purpose, the area that positively stained for each or both antibodies was measured in square pixels. The percentage of all FDC coexpressing CD23, CD35 or CD54, as assessed by the pan-FDC marker Ki-M4p, was calculated (\% co-expression). All values are expressed as mean with the standard deviation (SD) indicated.

For the correlation of FDC networks with clinical outcome in FL, we looked for a measure of the density of the FDC network within the follicles. For this the area 
covered by the FDC within 5 representative malignant follicles was quantified analogous to the double stainings and expressed as FDC covered area in relation to the area of the neoplastic follicle.

Statistical analysis

Co-expression was compared by means of t-tests using GraphPad prism (Graph Pad Prism Software Inc., San Diego, Ca, USA). To assess the prognostic impact of FDC networks on overall survival (OS) and treatment failure (TTF), Kaplan-Meier curves were calculated and group comparisons were done by means of the log rank test. The prognostic impact of numerical parameters was assessed by univariate Cox regression. 


\section{Results}

Expression of CD23, CD35 and CD54 is reduced in the dark zone of the GC

The density of the FDC network in the dark zone of the GC is known to be reduced, compared to the light zone. As a control of our fluorescence double staining method for quantifying FDC protein expression, we analyzed five GC in each of 10 hyperplastic tonsils. The dark zone of the GC was analyzed separately and compared to the entire GC, which is dominated by the dense FDC networks of the light zone. In all analyses, the area detected by the marker Ki-M4p was larger than the area staining for CD23, CD35 or CD54, indicating that Ki-M4p is useful as a panFDC marker ${ }^{12}$ (data not shown). Figure 1 shows representative examples of the staining pattern.

As shown in figure 2, the dark zone FDC displayed reduced co-expression of the FCreceptor/CD23 (8.5\%, SD 5.2), complement receptor 1/CD35 (20.2\%, SD 6.9) and ICAM/CD54 (19.0\%, SD 6.8), compared to the FDC in the entire follicle (CD23: 32.1\%, SD 10.8; CD35: 38.9\%, SD 15.7; CD54: $37.2 \%$, SD 10.8), and the differences were statistically significant $(p<0.0001, p=0.0029$ and $p=0.0003$, respectively, t-test). These data are in line with the published literature, which are based mainly on the analysis of mouse GCs and confirm our method for semi-quantitatively detecting protein expression in FDC.

\section{Expression of CD23, CD53 and CD54 is reduced in FDC in NHL compared to GC} Compared with the FDC in the GC as a whole, the FDC in FL, AITL and MCL displayed significantly reduced expression of CD23 ( $p=0.0012, p=0.0214$, and $p=0.0418$, respectively), CD35 ( $p=0.0067, p=0.002$, and $p=0.001$, respectively) and CD54 $(p=0.0017, p=0.0003$, and $p=0.0007$, respectively). Figure 3 provide a summary of all data. The level of co-expression of the above mentioned markers in the lymphomas was comparable to that of the FDC in the dark zone of the GC, with the exception of CD23, which was expressed at significantly higher levels in FDC of AITL and MCL than in the dark zone of the GC $(p=0.0031$, and $p=0,0054$ respectively, data not shown) 
The extent of FDC networks is not associated with clinical outcome in $\mathrm{FL}$

We quantified the FDC networks in malignant follicles in a previously characterized cohort of FL treated within a prospective randomized trial using single staining for the pan-FDC marker Ki-M4p or CD23. The area detected by the staining varied throughout the cohort examined for Ki-M4p (median 7.33 square pixels, minimum 2.52, maximum 24.48, $\mathrm{n}=102$ ) and CD23 (mean 10.86 square pixels, minimum 2.70, maximum 51.50, $n=81$ ). However, there was no association between the extent of the FDC network measured by Ki-M4p or CD23 with clinical outcome, as measured by time to treatment failure or overall survival (univariate and adjusted Cox regression, data not shown). 


\section{Discussion}

FDC are specialized antigen-presenting cells that are present exclusively in the GC 2. In normal GC, FDC interact with GC B cells via the immune complexes presented on FDC by FC-fragment binding receptors (CD23, CD32), complement receptors (CD35, CD21) and adhesion molecules (CD54, CD106, CD44) ${ }^{1}$. The proliferation of $B$ cells in the GC is stimulated by IL15 expressed on FDC ${ }^{1}$. Furthermore, survival signals are transmitted from FDC to proliferating GC B cells via BAFF/BLYS and Notch ligands DII1 and Jg1 ${ }^{13,14}$. Because of their close association and interaction with B cells, FDC are a key player in GC B cell proliferation, survival and selection ? In accordance with the function of the GC, the FDC network differs in the light and dark zones of the GC. The FDC in the light zone are predominantly involved in immune-complex presentation and selection of $B$ cells that produce high-affinity immunoglobulins directed against the antigen presented by the FDC. Thus, light zone FDC strongly express Fc fragment-binding proteins such as CD23 and CD32 and complement receptors such as CD21 and CD $35{ }^{3}$. The role of the FDC in the dark zone of the GC is less well defined. However, reduced expression of CD23 and CD21 has been noted, indicating that immune-complex presentation and selection of $B$ cells is not the predominant function of the FDC in the dark zone ${ }^{3}$. Instead dark zone FDC might be predominantly involved in the stimulation of proliferation and in the survival of expanding $B$ cells ${ }^{1,6}$.

That FDC exist in NHL has been known for a long time. In marginal zone lymphomas, the FDC networks might be considered to be remnants of pre-existing GC that have been overgrown and colonized by lymphoma cells. In other lymphoma entities such as FL, AILT and MCL, FDC networks are considered to be newly generated and to have proliferated during lymphoma expansion, although the FDC represent nonmalignant bystander cells that - most likely - do not belong to the lymphoma clone $^{7,15}$. The extent, distribution and morphologic shape of FDC networks in FL, AILT and MCL differ strikingly. In FL FDC networks are almost exclusively localized within malignant follicles and are absent in the interfollicular lymphoma compartment as well as in diffusely growing $\mathrm{FL}^{16}$. The most common morphological subtype of AITL (pattern 3) shows strikingly expanded and fused FDC networks with irregular shape ${ }^{17}$. Finally, in MCL the FDC networks are very variable in their extent, irregularly distributed and often only weakly developed ${ }^{18}$. 
Although the detection of FDC by immunohistochemical methods is a standard procedure in diagnostic hematopathology, the shape of FDC as cells with long dendritic extensions localized between $B$ cells makes it difficult to quantify the extent of the FDC network and to determine their immunohistochemical profile by conventional stainings. All published literature on FDC in NHL is based on conventional staining, examination by light microscopy and quantification by eyeballing on serial slides ${ }^{8,9,17}$. However, in recent years gene expression profiling studies have demonstrated that the expression of genes derived from non-lymphoma bystander cells such as $\mathrm{T}$ cells, macrophages and dendritic cells might be prognostically important ${ }^{10}$. Since gene expression profiling is not applicable to formalin-fixed, paraffin-embedded tissue (FFPE), alternative methods are required to quantify bystander cells. However, quantification of dendritic cells and their protein expression in FFPE tissues might not only be important for examining their possible role as prognostic markers but also for understanding the role of FDC in lymphomagenesis. In this study, we established a method for semiquantitative assessment of the extent of FDC networks and of protein expression in FDC. Our method, based on fluorescence double staining and digital image analysis, semiquantitatively reproduced the findings from the published literature with regard to the expression of CD23, CD35 and CD54 in the normal GC, which show reduced expression in the dark zone FDC ${ }^{3,8,9}$. Furthermore, we were able to demonstrate that the expression of all markers examined was also reduced in FDC found in FL, AITL and MCL. Our selection of FDC markers represents functional groups of FDC related proteins: Fc fragment-binding proteins for immune-complex presentation (CD23), complement receptors (CD35) and adhesion molecules (CD54) ${ }^{1}$. To some extent the immunophenotype of FDC in NHL resembles the FDC found in the dark zone of the $\mathrm{GC}^{3}$. Therefore, one might speculate that in FL, AITL and MCL FDC are not involved in immune-complex presentation and selection of $B$ cells but rather in the stimulation of proliferation and delivery of survival signals. Our findings are in contrast to earlier reports which suggested that FDC in FL of grade 1 or 2 were related to light zone FDC, based on the expression of $\mathrm{S} 100$ and caldesmon ${ }^{19}$. However, the proteins analyzed in this study are involved in key functions of FDC, and the expression pattern might indeed indicate a closer relation between FDC in NHL and dark zone FDC. 
Unfortunately, we were unable to establish reliable staining protocols for BAFF and IL15. Since the published literature on Baff expression by FDC show unexpected nuclear staining patterns ${ }^{13}$, we assume that reliable antibodies for FFPE tissues are currently not available. Baff and Notch ligands are expressed by FDC and are involved in mediating the survival and proliferation of $B$ cells ${ }^{13,14}$. Future studies will have to evaluate the expression of these proteins quantitatively in FDC of normal GC as well as NHL to understand whether the functions mediated by Baff and Notch ligands are intact in the FDC found in NHL.

Although our technique allows an accurate quantification of the extent of FDC networks within malignant follicles of $\mathrm{FL}$, we were unable to demonstrate an association between FDC networks and prognosis. Since gene expression signatures usually reflect complex biological processes involving the presence of multiple cell types, they might be difficult to surrogate by immunohistochemical stainings that detect one cell type only ${ }^{10,20}$. Prognostic gene expression signatures in FL might also reflect interactions of $\mathrm{T}$ cells with FDC, and this interaction might influence the expression of FDC proteins such as CD23 ${ }^{21}$. Furthermore, the shape of the FDC networks might also be of prognostic relevance and have not been assessed herein 22.

The question whether the specific lymphoma microenvironment develop by an infiltration of FDC and T-cells from outside or differentiation of the microenvironment in loco e.g. by stimuli provided by the NHL cells remains unsolved. Finally, a review of several contradictory studies on the prognostic impact of accessory cells like Tcells and FDC on prognosis in FL suggested that the effect might be dependent on the therapeutic protocols applied ${ }^{23}$. In summary, there seems little doubt about the important role of the microenvironment for the pathogenesis of NHL especially of chronic lymphocytic leukemia, FL or multiple myeloma ${ }^{24}$. However, transferring these basic research findings into clinical applicable bio-marker is difficult. Despite these difficulties, future studies using quantitative in situ methods like the one presented here will help us to understand which types of reactive bystander cell or which activation status of this cell type might be of use in the prognosis of NHL like FL. 


\section{Acknowledegements}

The authors would like to thank Olivera Batic and Monika Hauberg for their excellent technical assistance, Kay Dege for editing the manuscript and the colleagues from the reference centers in Würzburg, Lübeck and Frankfurt for contributing unstained slides.

\section{Authorship and disclosures}

WK was the principal investigator and takes primary responsibility for the paper. MD, WH recruited the patients. MKJ performed the laboratory work for this study. EH and MU participated in the statistical analysis. WK, EH wrote the paper. The authors reported no potential conflicts of interest. 


\section{References}

1. Park CS, Choi YS. How do follicular dendritic cells interact intimately with $B$ cells in the germinal centre? Immunology 2005;114;2-10.

Ref Type: Journal

2. Allen CD, Cyster JG. Follicular dendritic cell networks of primary follicles and germinal centers: phenotype and function. Semin. Immunol. 2008;20;14-25.

Ref Type: Journal

3. Yoshida K, van den Berg TK, Dijkstra CD. Two functionally different follicular dendritic cells in secondary lymphoid follicles of mouse spleen, as revealed by CR1/2 and FcR gamma IImediated immune-complex trapping. Immunology 1993;80;34-39.

Ref Type: Journal

4. Allen CD, Okada T, Cyster JG. Germinal-center organization and cellular dynamics. Immunity. 2007;27;190-202.

Ref Type: Journal

5. Allen CD, Okada T, Tang HL, Cyster JG. Imaging of germinal center selection events during affinity maturation. Science 2007;315;528-531.

Ref Type: Journal

6. Li L, Choi YS. Follicular dendritic cell-signaling molecules required for proliferation and differentiation of GC-B cells. Semin. Immunol. 2002;14;259-266.

Ref Type: Journal

7. Swerdlow SH, Campo E, Harris N et al. WHO Classification of Tumors of the Haematopoietic and Lymphoid Tissues. Lyon: IARC, 2008.

Ref Type: Book, Whole

8. Bagdi E, Krenacs L, Krenacs T, Miller K, Isaacson PG. Follicular dendritic cells in reactive and neoplastic lymphoid tissues: a reevaluation of staining patterns of $C D 21, C D 23$, and CD35 antibodies in paraffin sections after wet heat-induced epitope retrieval. Appl. Immunohistochem. Mol Morphol. 2001;9;117-124.

Ref Type: Journal

9. Chang KC, Huang X, Medeiros LJ, Jones D. Germinal centre-like versus undifferentiated stromal immunophenotypes in follicular lymphoma. J Pathol. 2003;201;404-412. Ref Type: Journal

10. Dave SS, Wright G, Tan B et al. Prediction of survival in follicular lymphoma based on molecular features of tumor-infiltrating immune cells. N. Engl. J. Med. 2004;351;2159-2169. Ref Type: Journal

11. Nickenig C, Dreyling M, Hoster E et al. Combined cyclophosphamide, vincristine, doxorubicin, and prednisone (CHOP) improves response rates but not survival and has lower hematologic toxicity compared with combined mitoxantrone, chlorambucil, and prednisone (MCP) in follicular and mantle cell lymphomas: results of a prospective randomized trial of the German LowGrade Lymphoma Study Group. Cancer 2006;107;1014-1022.

Ref Type: Journal

12. Parwaresch MR, Radzun HJ, Hansmann ML, Peters KP. Monoclonal antibody Ki-M4 specifically recognizes human dendritic reticulum cells (follicular dendritic cells) and their possible 
precursor in blood. Blood 1983;62;585-590.

Ref Type: Journal

13. Hase H, Kanno $\mathrm{Y}$, Kojima $\mathrm{M}$ et al. BAFF/BLyS can potentiate B-cell selection with the B-cell coreceptor complex. Blood 2004;103;2257-2265.

Ref Type: Journal

14. Yoon SO, Zhang X, Berner P, Blom B, Choi YS. Notch ligands expressed by follicular dendritic cells protect germinal center B cells from apoptosis. J Immunol. 2009;183;352-358.

Ref Type: Journal

15. Lennert K, Feller A. Histopathologie der Non-Hodgkin-Lymphome. Springer-Verlag Berlin Heidelberg New York, 1981.

Ref Type: Book, Whole

16. Klapper W, Hoster E, Rolver $L$ et al. Tumor sclerosis but not cell proliferation or malignancy grade is a prognostic marker in advanced-stage follicular lymphoma: the German Low Grade Lymphoma Study Group. J Clin. Oncol. 2007;25;3330-3336.

Ref Type: Journal

17. Attygalle A, Al Jehani R, Diss TC et al. Neoplastic T cells in angioimmunoblastic T-cell lymphoma express CD10. Blood 2002;99;627-633.

Ref Type: Journal

18. Schrader $C$, Meusers $P$, Brittinger $G$ et al. Growth pattern and distribution of follicular dendritic cells in mantle cell lymphoma: a clinicopathological study of 96 patients. Virchows Arch. 2006;448;151-159.

Ref Type: Journal

19. Tsunoda T, Yamakawa M, Takahashi T. Differential expression of $\mathrm{Ca}(2+)$-binding proteins on follicular dendritic cells in non-neoplastic and neoplastic lymphoid follicles. Am. J Pathol. 1999;155;805-814.

Ref Type: Journal

20. Staudt LM, Dave S. The biology of human lymphoid malignancies revealed by gene expression profiling. Adv. Immunol. 2005;87;163-208.

Ref Type: Journal

21. Sukumar S, Conrad DH, Szakal AK, Tew JG. Differential T cell-mediated regulation of CD23 (Fc epsilonRII) in B cells and follicular dendritic cells. J Immunol. 2006;176;4811-4817. Ref Type: Journal

22. Glas AM, Knoops L, Delahaye L et al. Gene-expression and immunohistochemical study of specific T-cell subsets and accessory cell types in the transformation and prognosis of follicular lymphoma. J Clin. Oncol. 2007;25;390-398.

Ref Type: Journal

23. de Jong D, Koster A, Hagenbeek A et al. Impact of the tumor microenvironment on prognosis in follicular lymphoma is dependent on specific treatment protocols. Haematologica 2009;94;70-77.

Ref Type: Journal

24. Burger JA, Ghia $P$, Rosenwald $A$, Caligaris-Cappio $F$. The microenvironment in mature B-cell malignancies: a target for new treatment strategies. Blood 2009;114;3367-3375.

Ref Type: Journal 


\section{Figure legends}

\section{Figure 1}

Representative example of fluorescence double staining using the pan-FDC marker Ki-M4p (green) and CD23 (red) in a normal germinal center (A). Co-expression of CD23 (B), CD35 (C) and CD54 (D) in representative examples of follicular lymphoma, AILT and MCL.

Figure 2

Quantitative analysis of co-expression of CD23, CD35 and CD54 on FDC in the entire germinal center (total GC) or the dark zone (DZ) in tonsils. P-values were obtained using ttests. Horizontal bars indicate the mean.

\section{Figure 3}

Quantitative analysis of co-expression of CD23 (A), CD35 (B) and CD54 (C) on FDC in the entire germinal center (total GC), follicular lymphoma (FL), angioimmunoblastic T-cell lymphoma (AITL) or mantle cell lymphoma (MCL). P-values were obtained using t-tests. Horizontal bars indicate the mean. 

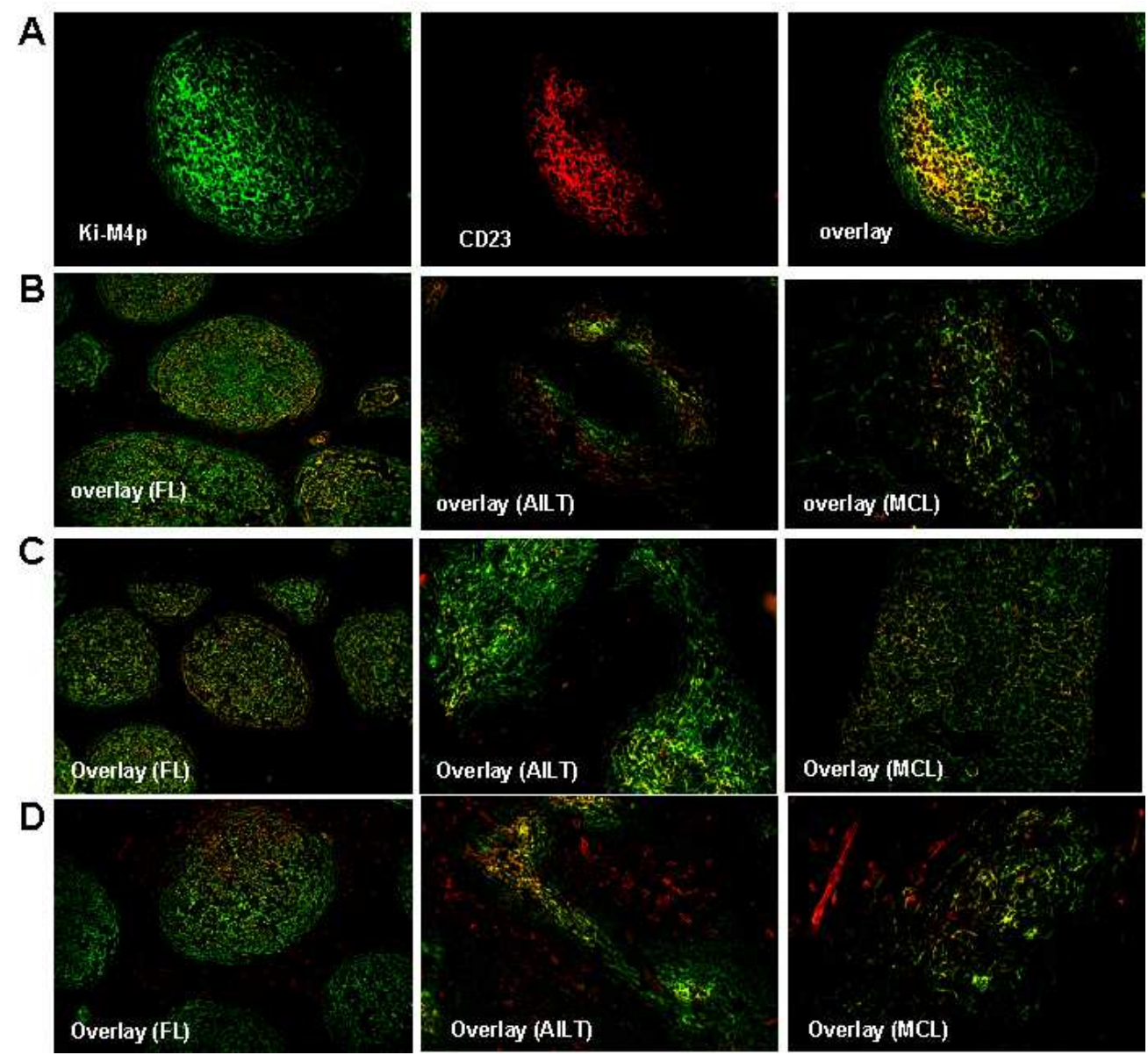

Representative example of fluorescence double staining using the pan-FDC marker Ki-M4p (green) and CD23 (red) in a normal germinal center (A). Co-expression of CD23 (B), CD35 (C) and CD54

(D) in representative examples of follicular lymphoma, AILT and MCL. $248 \times 227 \mathrm{~mm}(72 \times 72 \mathrm{DPI})$ 


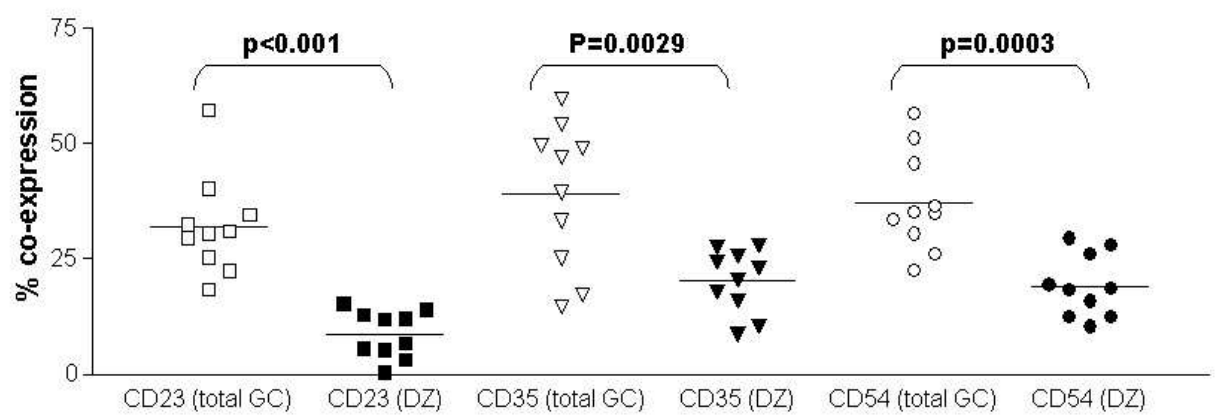

Quantitative analysis of co-expression of CD23, CD35 and CD54 on FDC in the entire germinal center (total GC) or the dark zone (DZ) in tonsils. P-values were obtained using t-tests. Horizontal bars indicate the mean.

$307 \times 103 \mathrm{~mm}(72 \times 72 \mathrm{DPI})$ 

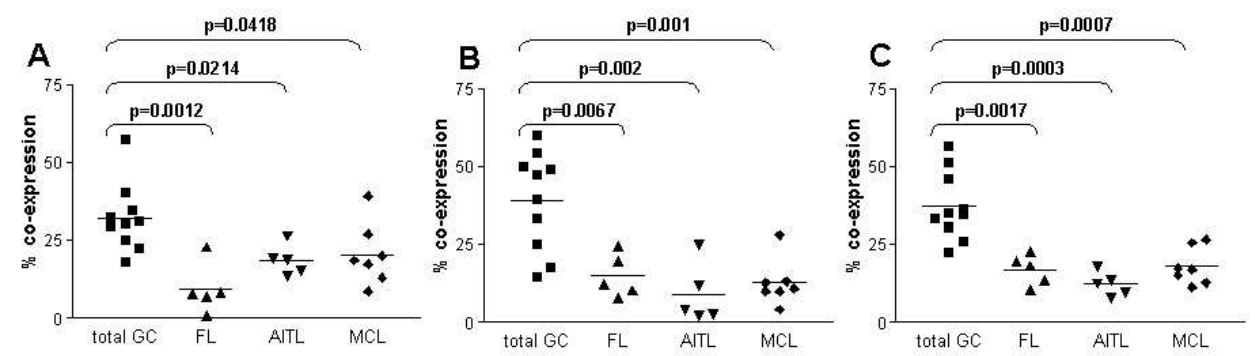

Quantitative analysis of co-expression of CD23 (A), CD35 (B) and CD54 (C) on FDC in the entire germinal center (total GC), follicular lymphoma (FL), angioimmunoblastic T-cell lymphoma (AITL) or mantle cell lymphoma (MCL). P-values were obtained using t-tests. Horizontal bars indicate the mean.

$337 \times 90 \mathrm{~mm}(72 \times 72 \mathrm{DPI})$ 